\title{
Human and canine pulmonary Mycobacterium bovis infection in the same household: re-emergence of an old zoonotic threat?
}

\author{
D Shrikrishna, ${ }^{1}$ R de la Rua-Domenech, ${ }^{2}$ N H Smith, ${ }^{3}$ A Colloff, ${ }^{4}$ I Coutts ${ }^{1}$
}

- Supplementary fig 2 is published online only at http:// thorax.bmi.com/content/vol64/ issue1

${ }^{1}$ Department of Respiratory Medicine, Royal Cornwall Hospital, Truro, UK; ${ }^{2}$ Bovine TB Programme, Department for Environment, Food and Rural Affairs (DEFRA), London, UK:

${ }^{3}$ Veterinary Laboratories Agency (VLA), Weybridge, UK and Centre for the Study of Evolution (CSE), University of Sussex, UK; ${ }^{4}$ Veterinary Laboratories Agency (VLA), Polwhele, Truro, UK

Correspondence to: Dr D Shrikrishna, Department of Respiratory Medicine, Royal Cornwall Hospital, Truro TR1

3LJ, UK; dinesh.shrikrishna@ nhs.net

Received 16 August 2008 Accepted 4 November 2008

\section{ABSTRACT}

Bovine tuberculosis (bTB), caused by Mycobacterium bovis, remains a serious animal health problem in the UK, despite longstanding statutory surveillance and control measures. Endemic infection in the Eurasian badger population is thought to complicate bTB eradication efforts. Sporadic cases of $M$ bovis infection have also been reported in domestic animals other than cattle. Human $M$ bovis infection is extremely rare in the native UK population in the absence of unpasteurised milk consumption or residence abroad. Here, pulmonary TB infection in a UK born female and her pet dog is described, caused by an identical strain of $M$ bovis. Latent TB infection was also identified in a household contact. The potential routes of infection and implications of this case are discussed.

\section{CASE NO 1: HUMAN INFECTION}

A 42-year-old UK born, Caucasian female living in Cornwall (South West of England, UK) presented in August 2007 with a 1 month history of general malaise and mucopurulent cough. Clinical examination was unremarkable but chest $x$ ray revealed right apical cavitation (fig 1A). Sputum was smear positive for acid fast bacilli and treatment was initiated with rifampicin, isoniazid, ethambutol and pyrazinamide. Subsequent culture grew Mycobacterium bovis and pyrazinamide was therefore withdrawn from the drug regimen. The isolated $M$ bovis strain underwent standard molecular typing at the Veterinary Laboratories Agency (VLA, Weybridge, UK) by spoligotyping and six loci variable number tandem repeats typing. ${ }^{1}$ The genotype recovered was SB0271: 7-45-4*-3-3.1; VLA genotype 12:a. The patient had no history of unpasteurised milk consumption, immunodeficiency or recent travel abroad. However, there was a history of potential occupational exposure to $M$ bovis when she worked as a veterinary nurse for two local practices in West Cornwall from 2000 to 2004. In particular, she assisted in tuberculin tests of cattle herds in the area and one farm that she visited had three skin test reactors in 2002, two of which were subsequently found to culture $M$ bovis at post mortem. The patient also recalled picking up an injured badger that had suffered a road traffic accident and transporting it to a veterinary surgery for treatment in 2004; this animal was euthanised a few days later without a postmortem examination. In addition, badgers were reportedly living in a sett at the bottom of the patient's garden.

\section{CASE NO 2: LATENT HUMAN INFECTION}

A household contact investigation, following the diagnosis of case No 1, identified that the patient's 12-year-old asymptomatic daughter had a positive Mantoux test with $16 \mathrm{~mm}$ induration. The daughter had not received the Bacille Calmette-Guérin (BCG) vaccination and, as in her mother's case, had no history of unpasteurised milk consumption, immunodeficiency or recent travel abroad. Clinical examination and chest $x$ ray were unremarkable, but subsequent blood testing using an interferon gamma release assay (QuantiFERON-TB Gold) was strongly positive and she was therefore initiated on chemoprophylaxis with rifampicin and isoniazid for latent tuberculosis (TB) infection. The other human household contacts screened did not show evidence of latent TB infection.

\section{CASE 3: CANINE INFECTION}

Four months after the initial presentation of case No 1, a pet dog in the household was noticed to have a persistent cough. Veterinary examination revealed increased respiratory noise on auscultation of the trachea. There was a partial response to treatment with enrofloxacin but the dog's condition then deteriorated and a sample of tracheal mucus was smear positive for acid fast bacilli. A presumptive diagnosis of mycobacterial infection was made and in agreement with the owner, the dog was euthanised. Macroscopic lesions at postmortem examination were confined to the respiratory tract and confirmed a granulomatous bronchitis, lymphadenitis and pneumonia (fig 2, see online data repository). Subsequent mycobacterial culture of the respiratory tract lesions grew $M$ bovis and molecular typing, as previously described, confirmed that the genotype was identical to that of the isolate from case No 1.

\section{DISCUSSION}

Despite the increasing incidence of bovine $\mathrm{TB}$ (bTB) in the UK since the mid-1980s, ${ }^{2} M$ bovis infections account for less than $1 \%$ of the laboratory confirmed cases of human $\mathrm{TB}$, in line with other industrialised countries..$^{3-5} M$ bovis infections in animals are transmissible to humans through inhalation of infectious aerosols, ingestion of unpasteurised dairy products or, less commonly, by contact with broken skin. ${ }^{5}{ }^{6}$ Historically, consumption of unpasteurised cows' milk accounted for the vast majority of human infection in the UK. ${ }^{5}$ However, with the almost universal adoption of milk pasteurisation, it has been suggested that inhalation, rather than ingestion, may now 


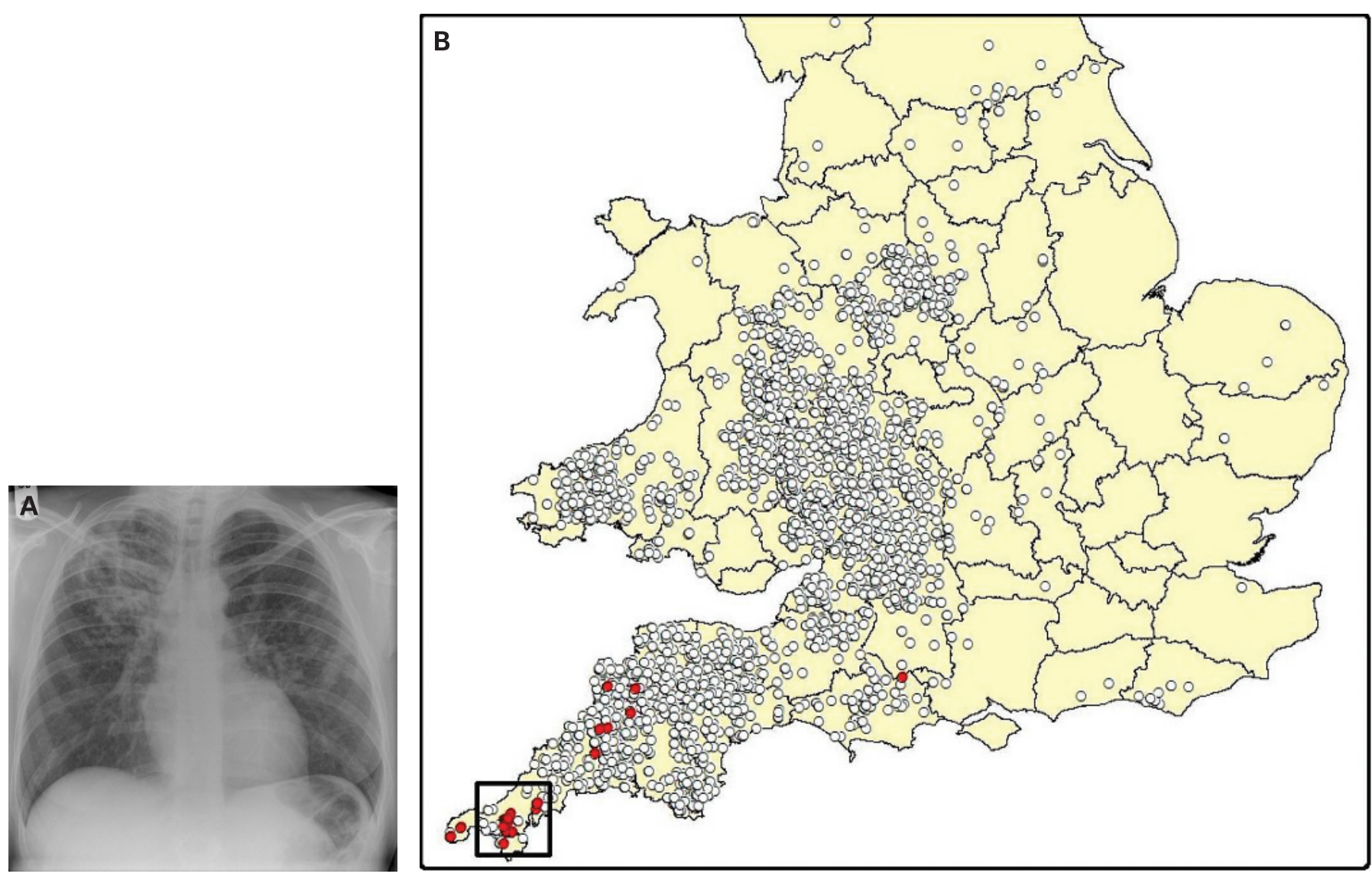

Figure 1 (A) Case No 1: chest radiograph showing right apical cavitation. (B) Map showing the location of all genotyped strains of Mycobacterium bovis from cattle in England and Wales in 2007 (white circles). Localisation of genotype 12:a strains to the South West of Cornwall (UK) from cattle in 2007 is shown (red circles). Over 40 isolates of 12:a from cattle were located within the black square, which marks the location of this case.

represent the predominant mode of $M$ bovis transmission from animals to humans. ${ }^{3}$ Although $M$ bovis has a broad host range, canine $M$ bovis infection is extremely rare in the UK, with only five cases confirmed by the VLA in the past 20 years. ${ }^{2} 7$

The genotype of $M$ bovis (12:a) recovered from case Nos 1 and 3 is relatively rare, occurring in less than $2 \%$ of the 50000

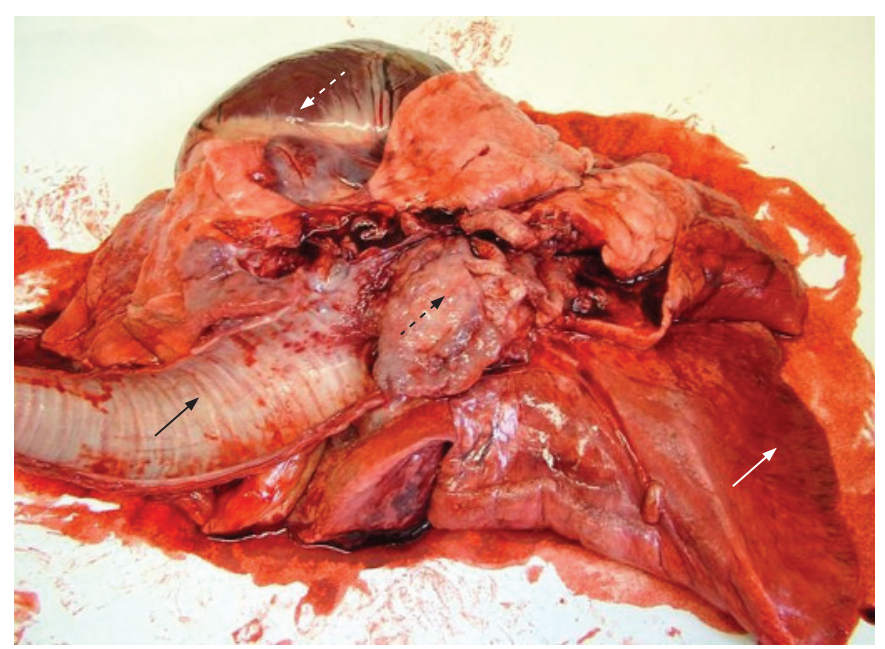

Figure 2 Case 3: canine postmortem specimen (see online data repository), trachea (black arrow), granulomatous lesion at tracheal bifurcation (black broken arrow), left lung (white arrow) and heart (white broken arrow). isolates from cattle recorded in the VLA Spoligotype Database. Isolates of this strain are geographically localised to cattle in the Southwest of Cornwall (fig 1B) and have not been reported outside of Great Britain to the authors' knowledge. ${ }^{1}$ All but five of the 46 badger samples with genotype 12:a recorded in the Spoligotype Database were also found in this immediate area. This indicates that the infections were, in all likelihood, locally acquired. As case No 1 was a middle-aged person and had no history of unpasteurised milk consumption, the evidence points to a recent, native $M$ bovis infection, probably as a consequence of airborne exposure to an infectious animal or its excretions. The original source of infection for the two confirmed cases (human and canine) may never be definitively elucidated, but it is reasonable to assume direct or indirect transmission from cattle or badgers.

There are several hypotheses as to the source of infection in the affected household. The chronology of clinical disease suggests that case No 1 may have been the index case, with subsequent airborne transmission of infection to her daughter and pet dog. Person to person transmission of $M$ bovis infection is extremely rare, but has been recently reported among immunocompromised $^{8}$ and immunocompetent ${ }^{9}{ }^{10}$ patients. Reverse zoonotic transmission of $M$ bovis and $M$ tuberculosis from humans to animals is also rare, but has been described previously. ${ }^{6}{ }^{11}$ The source of infection for case No 1 may have been through previous occupational exposure to $M$ bovis infected animals while working as a veterinary nurse. Although there was no history of immunodeficiency to suggest reactivation of latent infection, interestingly one of the cattle 
herds that she helped to tuberculin test in 2002 was found to be infected with spoligotype 12. Unfortunately, no variable number tandem repeats data are available for this herd but spoligotype 12, when subtyped, is almost invariably of genotype 12:a, the local strain of $M$ bovis. We have no reports of TB being detected in either the attending veterinarian or the herd keeper, both of whom would have had much closer and prolonged contact with any infected cattle. Nevertheless, this infected cattle herd does represent a potential source of infection for case No 1 . It is also possible that case 1 could have been infected through contact with badgers, both on her property and as part of her previous work, particularly as badgers have been identified as a maintenance host of $M$ bovis. ${ }^{12}$

An alternative possibility is that the tuberculous pet dog acquired $M$ bovis infection from a local animal reservoir and then infected the human contacts through airborne transmission. $M$ bovis infection does occur sporadically in companion animals living in rural and suburban areas and this is thought to represent spillover of infection from cattle and wildife reservoirs. ${ }^{73}$ However, even though the actual onset of the dog's pulmonary infection cannot be reliably established, the fact that its clinical signs were noticed 4 months after the owner presented with cavitating disease suggests that the canine to human route of transmission was less likely.

Finally, there is a small possibility that all three cases independently acquired $M$ bovis infection from a common animal source. The household is located within an area of high bTB incidence and, therefore, the local cattle and badger populations would represent a potential common source of exposure.

It has recently been suggested that since the introduction of milk pasteurisation, $\mathrm{bTB}$ poses a negligible public health risk in the UK. ${ }^{14}$ However, this case demonstrates that human infection with $M$ bovis acquired in the UK is not a disease of the past. The isolation of an identical genotype of $M$ bovis from a pet dog and its owner, in a rural area where the same strain is often recovered from cattle and badgers, identifies a low, but ongoing, public health risk. The potential for airborne transmission of $M$ bovis, particularly to segments of the rural population, highlights the need for vigilance and effective collaboration between clinicians, animal and public health professionals in the management of this often forgotten infection.

Acknowledgements: The authors gratefully acknowledge the contribution of Jo Wheeler, Veterinary Officer, Animal Health Divisional Office, Truro, UK.

Competing interests: None.

Patient consent: Patient consent was obtained.

\section{REFERENCES}

1. Smith NH, Gordon SV, de la Rua-Domenech R, et al. Bottlenecks and broomsticks: the molecular evolution of Mycobacterium bovis. Nat Rev Microbiol 2006:4:670-81.

2. Anon. Animal Health 2007: The report of the Chief Veterinary Officer. London: Defra Publications 2008:34-40. http://www.defra.gov.uk/animals/cvo/report.htm (accessed 11 November 2008)

3. Jalava $\mathbf{K}$, Jones JA, Goodchild $\mathrm{T}$, et al. No increase in human cases of Mycobacterium bovis disease despite resurgence of infections in cattle in the United Kingdom. Epidemiol Infect 2007;135:40-5.

4. Thoen C, LoBue P, de Kantor I. The importance of Mycobacterium bovis as a zoonosis. Vet Microbiol 2006:112:339-45.

5. de la Rua-Domenech R. Human Mycobacterium bovis infection in the United Kingdom: Incidence, risks, control measures and review of the zoonotic aspects of bovine tuberculosis. Tuberculosis (Edinb) 2006;86:77-109.

6. O'Reilly LM, Daborn CJ. The epidemiology of Mycobacterium bovis infections in animals and man. Tuber Lung Dis 1995;76:S1-46.

7. Ellis MD, Davies S, McCandlish IA, et al. Mycobacterium bovis infection in a dog. Vet Rec 2006;159:46-8.

8. Evans JT, Grace Smith E, Banerjee A, et al. Cluster of human tuberculosis caused by Mycobacterium bovis: evidence for person-to-person transmission in the UK. Lancet 2007;369:1270-6.

9. LoBue PA, Betancourt W, Cowan L, et al. Identification of a familial cluster of pulmonary Mycobacterium bovis disease. Int J Tuberc Lung Dis 2004;8:1142-6.

10. Smith RM, Drobniewski F, Gibson A, et al. Mycobacterium bovis infection, United Kingdom. Emerg Infect Dis 2004;10:539-41.

11. Fritsche A, Engel R, Buhl D, et al. Mycobacterium bovis tuberculosis: from animal to man and back. Int J Tuberc Lung Dis 2004:8:903-4.

12. Donnelly CA, Wei G, Johnston WT, et al. Impacts of widespread badger culling on cattle tuberculosis: concluding analyses from a large-scale field trial. Int $\mathrm{J}$ Infect Dis 2007:11:300-8.

13. Monies R, Jahans K, de la Rua-Domenech R. Bovine tuberculosis in cats. Vet Rec 2006;158:245-6.

14. Torgerson $\mathbf{P}$, Torgerson D. Does risk to humans justify high cost of fighting bovine TB? Nature 2008; $455: 1029$. 
Corrections

doi:10.1136/thx.2008.102947

O'Driscoll B R, Howard L S, Davison A G on behalf of the British Thoracic Society. BTS guideline for emergency oxygen use in adult patients. Thorax 2008; 63(Suppl 6):vi1-vi68.

The Guideline Development Group regret that no advice was offered for the management of status epilepticus. As this is a life-threatening condition where a patient may suffer from cerebral hypoxia (and oximetry may not be possible), patients with status epilepticus should be treated in accordance with table 1 (reservoir mask until clinically stabilised). This advice also applies to other rare conditions that may cause life-threatening hypoxaemia that are not listed specifically in table 1.

Roberts JA. Benchmarking chronic obstructive pulmonary disease across an inner city primary care trust: one year on. Thorax 2008;63(Suppl vii):A8 (abstract S12).

The correct authors for this abstract are as follows: Roberts JA, Bakerly ND.

Roberts JA. Should chronic obstructive pulmonary disease service delivery in an inner city primary care trust be targeted at general practice or practice-based commissioning cluster level? Thorax 2008;63(Suppl vii):A8 (abstract S13).

The correct authors for this abstract are as follows: Roberts JA, Bakerly ND.

Tsartsali L, Fleming L, Regamey N, et al. Relationship between non-invasive inflammatory markers and the current level of clinical control in childhood asthma. Thorax 2008;63(Suppl vii):A33 (abstract S70).

This abstract has been withdrawn.

Baird S, Ashish A, O'Connor J, et al. Respiratory assessment centre: does it increase the number of patients taken home with the early supported discharge team? Thorax 2008;63(Suppl vii):A67 (abstract S154).

It has come to the attention of the Scientific Committee that this abstract was not seen by all the authors prior to submission and is therefore withdrawn.

doi:10.1136/thx.2007.088831

Creagh-Brown B C, Nicholson A G, Showkathali R, et al. Pulmonary veno-occlusive disease presenting with recurrent pulmonary oedema and the use of nitric oxide to predict response to sildenafil Thorax 2008;63:933-4.

The address for correspondence should be: Dr B C Creagh-Brown, Adult Intensive Care Unit, Royal Brompton Hospital, London SW3 6NP, UK; drbencb@gmail.com. 